\title{
REVIEW ARTICLE Point-of-care lung ultrasound in neonatology: classification into descriptive and functional applications
}

\author{
Francesco Raimondi ${ }^{1}$, Nadya Yousef ${ }^{2}$, Fiorella Migliaro ${ }^{1}$, Letizia Capasso ${ }^{1}$ and Daniele De Luca ${ }^{3}$
}

\begin{abstract}
Lung ultrasound (LUS) is the latest amongst imaging techniques: it is a radiation-free, inexpensive, point-of-care tool that the clinician can use at the bedside. This review summarises the rapidly growing scientific evidence on LUS in neonatology, dividing it into descriptive and functional applications. We report the description of the main ultrasound features of neonatal respiratory disorders and functional applications of LUS aiming to help a clinical decision (such as surfactant administration, chest drainage etc). Amongst the functional applications, we propose SAFE (Sonographic Algorithm for liFe threatening Emergencies) as a standardised protocol for emergency functional LUS in critical neonates. SAFE has been funded by a specific grant issued by the European Society for Paediatric Research. Future potential development of LUS in neonatology might be linked to its quantitative evaluation: we also discuss available data and research directions using computer-aided diagnostic techniques. Finally, tools and opportunities to teach LUS and expand the research network are briefly presented.
\end{abstract}

Pediatric Research (2021) 90:524-531; https://doi.org/10.1038/s41390-018-0114-9

\section{INTRODUCTION}

The first report on the use of lung ultrasonography (LUS) in adult medicine appeared in $1995^{1}$ and LUS has been rapidly gaining popularity, also in paediatrics and in neonatology. LUS is a point-ofcare, easy-to-learn, radiation-free, bedside, quick and repeatable technique. LUS signs vary little by age, ${ }^{2}$ which makes it especially suitable for use in the smallest patients and in the critical care setting. In the past 10 years, there has been a notable increment in publications on the use of LUS in neonatology (Fig. 1), and even more in adult medicine. We demonstrated that launching a LUS program in their neonatal intensive care unit (NICU) roughly halved the number of chest radiograms and significantly decreased the mean radiation dose/patient. ${ }^{3}$ We present a comprehensive review on LUS in neonatology with an emphasis on pathophysiology and on a classification into descriptive (qualitative) and functional (semiquantitative) applications.

\section{DESCRIPTIVE LUNG ULTRASOUND}

LUS is a powerful diagnostic technique and a noninvasive research tool to describe several neonatal respiratory disorders in a qualitative manner. International LUS guidelines for adult critical care include a short chapter about applications in infants, based on the little data available in 2012, and conclude that the use of descriptive LUS may be of interest. ${ }^{4}$ Many studies have been published since then, and we aim to review the knowledge available today. The main LUS semiology patterns are illustrated in Fig. 2 and in the supplementary material S1-S5. The description of LUS findings for each neonatal lung disorder is summarised in Table 1.
Transient tachypnoea of the neonate

The main pathophysiological feature of transient tachypnoea of the neonate (TTN) is delayed lung fluid re-absorption during the foetal life transition and this creates a mainly interstitial, ab extrinseco lung oedema. LUS shows a high sensitivity and specificity to detect alveolar-interstitial oedema and to estimate extravascular lung water (EVLW) in adults through the evaluation of B-lines, which are vertical dynamic artefacts arising at the fluid/ air interface. ${ }^{5,6}$ B-lines may be sparse or confluent, creating a continuum that is generally referred to as an 'alveolar-interstitial pattern'.

Using LUS, substantial liquid retention has been demonstrated at 10 years of life in $14 \%$ of healthy neonates, while $49 \%, 78 \%$ and $100 \%$ of neonates had completed airway liquid clearance at 2, 4 and $24 \mathrm{~h}$, respectively. ${ }^{7}$ Moreover, it seems that neonates born by an elective caesarean section have higher fluid retention early after birth than those vaginally delivered. ${ }^{7-9}$ Consistently, Copetti and Cattarossi showed that neonates with TTN have both interstitial oedema (represented by B-lines), and normal areas (represented by A-lines). A sharp increase in echogenicity was described in the lower lung fields of TTN neonates and the authors named this finding 'double lung' point. ${ }^{10}$ Subsequent publications showed that the double lung point is not a perfect diagnostic tool for TTN, whose ultrasound appearance may include pleural line thickness, a more diffused alveolar-interstitial pattern and the presence of normally aerated areas. ${ }^{11,12}$ This seems to be confirmed by the preliminary data of an ongoing multicentre, international study. ${ }^{13}$ Despite the rich semiology and the absence of a unique diagnostic sign, the distinction between TTN and respiratory distress syndrome (RDS) is relatively easy (see below),

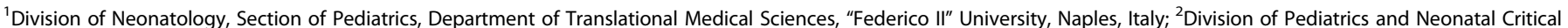

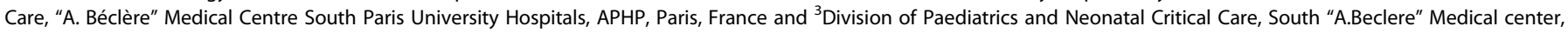
South Paris University Hospitals, APHP and South Paris-Saclay, Paris, France

Correspondence: Francesco Raimondi (raimondi@unina.it)

These authors contributed equally: Francesco Raimondi, Nadya Yousef

Received: 13 March 2018 Revised: 28 May 2018 Accepted: 20 June 2018

Published online: 20 July 2018 
but it should be kept in mind that clinical and laboratory data must always be integrated with LUS to refine the diagnosis. ${ }^{14}$

Respiratory distress syndrome

The typical LUS appearance of respiratory distress syndrome (RDS) consists of bilateral white lungs (i.e., the diffuse alveolar-interstitial pattern) with no spared areas reflecting decreased air/fluid ratio. Other findings include 'sub-pleural' small consolidations and/or an irregular pleural line. ${ }^{11}$ These results have been confirmed by multiple studies. ${ }^{11-13,15-17}$ Since RDS is a more severe and diffuse condition than $\mathrm{TTN}$, the absence of spared areas (with A-lines) seems the most noticeable sign and, contrary to $\Pi \mathrm{NN}$, in the absence of any treatment, LUS appearance will not improve

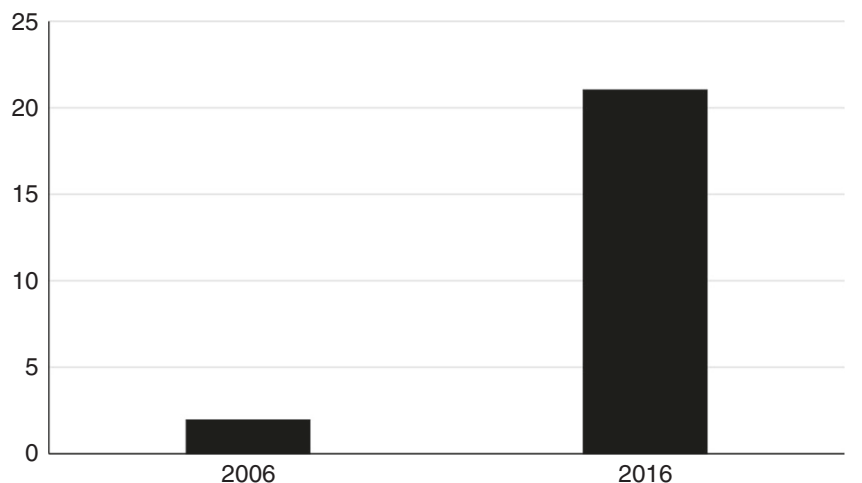

Fig. 1 Papers published on lung ultrasound in neonatology in 2006 and 2016. Retrieved by searching in PubMed (on 30 Dec 2017), limited to the newborn age, with the following words and MeSH terms: (("lung"[MeSH Terms]OR"lung"[All Fields])AND ("diagnostic imaging"[Subheading]OR("diagnostic"[All Fields]AND“imaging"[All Fields]) OR"diagnostic imaging"[All Fields]OR"ultrasound"[All Fields]OR"ultrasonography"[MeSH Terms]OR "ultrasonography"[All Fields]OR"ultrasound"[All Fields]OR"ultrasonics"[MeSH Terms]OR"ultrasonics"[All Fields])) AND((“2006/01/01"[PDAT]: "2006/12/31"[PDAT])AND "infant, newborn"[MeSH Terms])OR(("2016/01/01"[PDAT]:"2016/12/31"[PDAT]) quickly. ${ }^{11}$ There is a high inter-observer agreement among physicians with different levels of LUS expertise, which makes the differential diagnosis between RDS and TTN reliable, irrespective of the operator. ${ }^{18}$ LUS is also useful in diagnosing the complications of RDS, such as pulmonary haemorrhage, pneumothorax (PNX) or atelectasis. ${ }^{19,20}$

Two pathophysiological aspects must be considered: (1) clinical and laboratory data must always be integrated with LUS to refine the diagnosis of RDS, especially when it may coexist with other conditions, like pneumonia, early-onset sepsis or air leaks; ${ }^{15}$ (2) mixed-type situations may exist where fluid retention is associated with partial surfactant deficiency, as this has been recently demonstrated by lamellar body count. ${ }^{21}$ Interestingly, a semiquantitative LUS score describing lung aeration (see below) correlated with lamellar body count. ${ }^{22}$ These mixed TTN/RDS cases may last longer than classical TTN and may sometimes require noninvasive respiratory support or even a surfactant. ${ }^{23}$

Unlike chest X-rays, LUS appearance does not change shortly after surfactant administration, ${ }^{24}$ and this is intrinsically due to their different properties: LUS detects lung fluid content, while Xrays directly detect lung aeration. Surfactant replacement unavoidably implies some fluid administration even with the more concentrated surfactant preparations. Animal data demonstrate an almost total EVLW clearance $6 \mathrm{~h}$ after surfactant administration. ${ }^{24}$ However, the same process seems more variable and heterogeneous in human neonates, as LUS appearance may be influenced by respiratory support, gestational age, fluid intake, pre-existing condition (pure RDS or a more complex situation with superimposed lung inflammation and surfactant catabolism, such as acute respiratory distress syndrome (ARDS)) and the eventual simultaneous development of broncho-pulmonary dysplasia (BPD).

\section{Neonatal ARDS}

ARDS is an acute, life-threatening respiratory failure, characterised by extensive lung tissue inflammation, endothelial injury and both quantitative and qualitative secondary surfactant dysfunction, leading to loss of lung aeration. ${ }^{25}$ Neonatal ARDS shares the same biological and pathophysiological aspects of the syndrome in 
Table 1. Descriptive lung ultrasound findings in different neonatal lung disorders

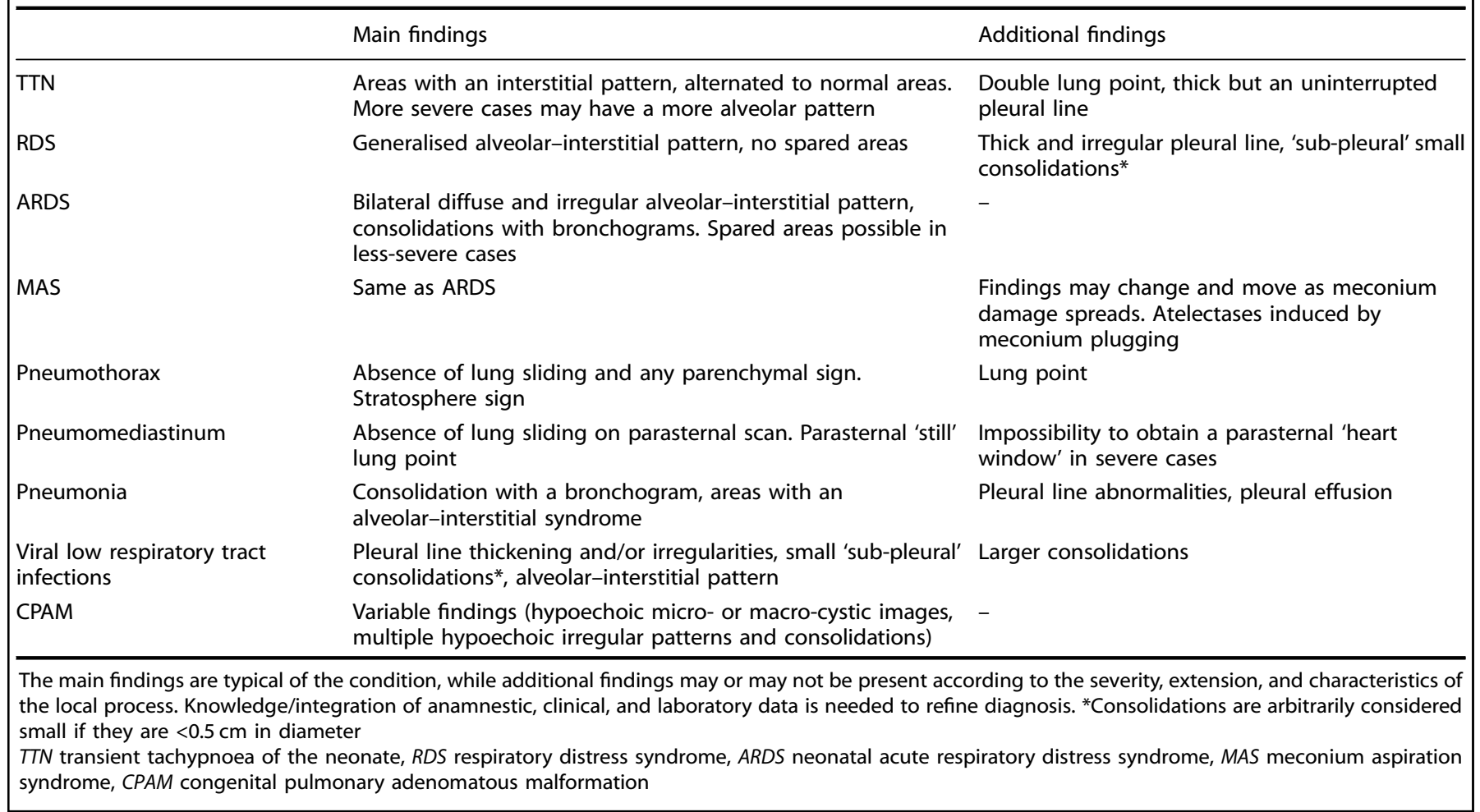

older patients: thus, signs on lung imaging are similar. LUS findings in neonatal ARDS consist of bilateral diffuse loss of aeration, which may vary from a diffuse alveolar-interstitial to an irregular alveolar pattern with consolidations with bronchograms and/or atelectases. ${ }^{26}$

Lung imaging is one of the diagnostic criteria included in the Montreux definition of neonatal ARDS, but this officially requires $X$-ray findings (diffuse, bilateral and irregular opacities or infiltrates, or complete opacification of the lungs, which are not fully explained by local effusion, atelectasis, RDS, TTN or congenital lung anomalies). ${ }^{25}$ Nonetheless, LUS has been used for the diagnosis of ARDS in adults ${ }^{27}$ and is considered suitable in neonates if sufficient clinical expertise exists for its interpretation. $^{28}$

Despite similarities with the syndrome in older patients, neonatal ARDS may also have different triggers, such as meconium aspiration syndrome (MAS), lung haemorrhage, perinatal asphyxia or necrotising enterocolitis that are peculiar to newborn age.

\section{Meconium aspiration syndrome}

Meconium aspiration syndrome (MAS) is the only ARDS-triggering condition for which LUS findings have been formally described so far and they consist of a mix of normal lung areas, coalescent or sparse alveolar-interstitial pattern and consolidations with bronchograms. These signs are irregularly present all over the lungs and may change over time as the meconium-driven inflammation progresses; meconium plugs may also occur and create atelectases. ${ }^{29}$ These findings were confirmed in a larger study of 117 neonates with MAS and a dissociation between clinical severity and imaging findings may sometimes occur. ${ }^{30}$ In summary, LUS signs in MAS include all the possible findings ranging from normally aerated zones to a complete loss of aeration, and, when the injury is sufficiently severe and diffuse, the lesions may cause an important oxygenation impairment and qualify as neonatal ARDS.
Air leak syndromes

LUS signs of PNX (see Supplementary material S4 and S5) are the absence of lung sliding and of any other sign other than A-lines; these findings are described in detail elsewhere. ${ }^{31}$ LUS has a higher diagnostic accuracy than conventional radiology for the diagnosis of PNX in adults, as it has been demonstrated by a metaanalysis of 13 studies. ${ }^{32}$ Therefore, LUS can potentially detect subclinical PNX that may go radiologically underdiagnosed and that does not require treatment. Recently, a case report and two diagnostic accuracy studies suggest that LUS may also be very useful in the diagnosis of neonatal PNX. ${ }^{33-35}$ In critically ill babies, LUS can be used for rapid detection of life-threatening tension PNX: an international multicentre study confirmed that LUS has an optimal diagnostic accuracy and is quicker than conventional radiology. ${ }^{36}$ LUS also resulted more accurately than chest transillumination (which is also less accurate than conventional radiology). ${ }^{34}$

A case report described the use of LUS to detect and follow up neonatal interstitial emphysema. ${ }^{37}$ No formal LUS description of neonatal pneumomediastinum exists. However, pneumomediastinum has been detected in children as (1) the absence of lung sliding on parasternal scan (with normal sliding in other chest areas); (2) a parasternal 'still' lung point, since the air collection displaces the lungs laterally and reveals the border between the air-filled mediastinum and the displaced lung; this still lung point does not move with spontaneous breathing and it remains under the parasternal area; (3) impossibility to obtain a normal parasternal heart view due to air artefact, regardless of the

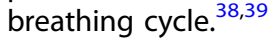

\section{Pneumonia}

LUS typically shows pneumonia as the presence of consolidations with irregular borders and air bronchograms, associated with pleural line abnormalities, and alveolar-interstitial pattern in the adjacent areas if the inflammatory process is extended. 
The presence of these signs carries an optimal diagnostic accuracy according to a study performed on 40 neonates with symptomatic pneumonia and 40 controls. ${ }^{40}$ Similar results were obtained in a larger cohort of 3405 Chinese neonates, of whom 725 were diagnosed with pneumonia by routine LUS: among 81 cases without any sign of lung disease by chest radiograms, there were 32 cases with clinical and ultrasound evidence of pneumonia. ${ }^{41}$ These data are fully consistent with those obtained in older patients. In fact, a meta-analysis of eight diagnostic studies (765 paediatric patients, including both neonates and children) yielded a sensitivity and a specificity of 96 and 93\%, respectively, which is superior to the accuracy of chest radiograms and comparable to that obtained combining radiology and laboratory exams. ${ }^{42}$ Similarly, a meta-analysis of 20 studies (2513 adults) showed that LUS has a high accuracy (sensitivity $85 \%$, specificity $93 \%$ ) to diagnose pneumonia defined by the combination of radiological and clinical data. ${ }^{43}$ Finally, a smaller metaanalysis of 12 studies (1515 adults) showed LUS to be more accurate than conventional radiology or computerised tomography $(\mathrm{CT})$ alone. $^{44}$

Some points still deserve to be investigated. There are no specific data regarding pneumonia of different types (i.e. congenital, community-acquired or ventilator-associated), although lobar or haemilobar consolidations are useful to diagnose ventilatorassociated pneumonia in adults, when coupled with clinical diagnostic criteria. ${ }^{45}$ Thresholds for the size of consolidations and exact measurement methods also need to be defined.

Bronchiolitis and other viral low tract respiratory infections Bronchiolitis, often caused by respiratory syncytial virus (RSV), is essentially an airway inflammatory disease causing obstructive respiratory failure with possible secondary involvement of the alveolar tissue. Therefore, from an ultrasound point of view, bronchiolitis presents as a nonhomogeneous pleural line abnormalities (pleural line thickening and/or irregularities), small 'subpleural' and/or larger consolidations or an alveolar-interstitial pattern, in the case of parenchymal involvement. ${ }^{46}$ For the sickest patients, consolidations may span across several intercostal spaces, ${ }^{47}$ due to associated atelectasis, viral alveolar injury or superimposed bacterial infection. If the lung injury is severe enough, patients may qualify for RSV-induced ARDS and this is characterised by a shift towards a mainly restrictive and severe respiratory failure ${ }^{48}$ with a greater loss of aeration, as described above. LUS findings correlate with disease severity, with a higher proportion of hospitalised patients having positive findings compared to outpatients. ${ }^{49}$ Moreover, findings gradually resolve with clinical improvement and the lung aeration correlates with the duration of oxygen therapy both in spontaneously breathing infants ${ }^{50}$ and in those needing noninvasive respiratory support. ${ }^{47}$ A good concordance among operators of different expertise has been reported for the ultrasound evaluation of bronchiolitis, ${ }^{49}$ similar to that reported for restrictive disorders. ${ }^{18}$

Bronchiolitis findings are non-specific and shared with other viral low tract respiratory infections; thus, laboratory tests are warranted to clarify the aetiology and also rule out bacterial coinfection. ${ }^{51}$ Only one study has evaluated LUS during a H1N1 outbreak suggesting moderate accuracy in distinguishing viral and bacterial pneumonias, as these were showing an interstitial pattern and consolidations, respectively. ${ }^{52}$ However, this study was performed during an outbreak and may be biased by the high disease prevalence. Consistently, other case series in adults have shown nonspecific LUS findings in influenza and measles. ${ }^{53-55}$ No specific neonatal studies are available in this field.

\section{Broncho-pulmonary dysplasia}

Early prediction of worsening respiratory conditions and bronchopulmonary dysplasia (BPD) is a potentially interesting application for LUS, as other techniques have failed in this regard. ${ }^{5,57}$ Two studies published in the nineties addressed LUS features of BPD using the trans-abdominal approach. The authors found that in BPD-developing babies, LUS showed the persistence or the appearance of nonhomogeneous retro-diaphragmatic hyperechogenicity, which was not visible in controls. ${ }^{58,59}$. Since then, ultrasound technology and our understanding of BPD have greatly improved. Data on transthoracic LUS and BPD are currently lacking. Moreover, there are no LUS data describing 'developing BPD' or the new concept of chronic pulmonary insufficiency of prematurity, that may provide new interesting areas of application for LUS. 60

\section{Malformations}

LUS has been used to describe congenital pulmonary airway malformations (CPAM), which have a variable appearance (a large or micro-cystic lesion or irregular consolidations) in line with the four histological types described in the most recent CPAM classification. $^{61,62}$ The gold standard to diagnose lung malformations remains the CT-scan, though LUS may allow to suspect CPAM in the absence of an antenatal diagnosis. ${ }^{62}$

\section{FUNCTIONAL LUNG ULTRASOUND}

LUS may also be used in a more 'functional' way, to guide therapeutic interventions or to assist during invasive procedures. Some examples of these applications already exist in the form of LUS scores or decision-making protocols in adult critical care. ${ }^{63,64}$ We shall review a few neonatal data and provide specific proposals for these applications in neonatology.

\section{Scores for semi-quantitative LUS}

A basic, three-stage classification can be set as coalescent $B$ lines (i.e. the 'white lung image'), sparse B lines and the normal, diffuse A-line pattern. Using this simple system, our group monitored the postnatal lung fluid clearance and predicted NICU admission in a cohort of late preterm and term infants. ${ }^{65}$ This information may be particularly valuable to healthcare providers in level I/II perinatal centres. We also investigated the usefulness of LUS linking a specific LUS profile to a therapeutic decision: the presence of a bilateral severe alveolar-interstitial pattern reliably predicted the need for intubation in NICU-admitted preterm neonates (sensitivity $88.9 \%$, specificity $100 \%) .{ }^{66}$ Similar results were found by other authors in a cohort of neonates older than 32 weeks, arbitrarily classifying the LUS findings as low (normal or TTN) or high risk (RDS, MAS, pneumothorax or pneumonia). ${ }^{67}$

These papers used descriptive LUS to predict or guide clinical decision but did not quantify the LUS findings. Since LUS detects the artefacts generated by the accumulation of fluid, and given that artefacts may be ranked according to the air/fluid ratio, it is possible to create scores inversely reflecting lung aeration. Several semi-quantitative scores are available in adult critical care and their description is beyond our scope. Nonetheless, all LUS scores are based on the same semiology and, interestingly, they seem only useful in restrictive lung disorders. In fact, an obstructive condition will create air-trapping and this might not be distinguishable from a normally aerated lung at LUS, as both present with A-lines. Consistently, LUS scores may well evaluate lung aeration, but they cannot detect over-distension, as it has been proven in ventilated adults. ${ }^{68}$ Despite these limitations, LUS scores offer the advantage of allowing serial semi-quantitative evaluations of the disease severity.

We described the first LUS score to be used in neonates with respiratory failure modifying a score already used in adult critical care. ${ }^{69}$ The main modifications were (1) fewer lung areas to scan given the smaller chest size; (2) use of a small linear or a microlinear 'hockey-stick' probe instead of a convex one. We were able to demonstrate that the LUS score is suitable and inversely correlated to oxygenation. ${ }^{70}$ The score is based on three chest 
areas for each side (upper anterior, lower anterior and lateral) and a 0-to-3 score is given for each area: more details are shown in supplementary material S6. The LUS score is able to predict the need for surfactant treatment in preterm infants below 34 weeks' gestation (area under the curve: 0.93 (95\% Cl: 0.86-0.99; $p<$ $0.001)){ }^{70}$ and in extremely preterm neonates affected by RDS (area under the curve: 0.94 (95\% Cl: $0.90-0.98 ; p<0.001)$ ). ${ }^{11}$ The diagnostic accuracy was lower in late preterm and term neonates since they may be affected by various lung disorders with different appearance and severity, such as RDS and TTN, but also aspiration syndromes and sepsis or ARDS. LUS score calculation has a high inter-observer agreement regardless of the ultrasonographers' experience. $^{70}$

Computer-aided image analyses provide an appealing approach for interpreting LUS and different technologies are being developed. ${ }^{72}$ For instance, ultrasound lung texture analysis has already been used to examine the foetal lung and predict the need of respiratory support. ${ }^{73}$ We found a significant correlation between the LUS score calculated by the ultrasonographer or by a supervised machine-learning approach and oxygenation indexes, while a LUS score obtained with greyscale analysis, another computerised image analysis technique, did not correlate with oxygenation. ${ }^{74}$ Computer technology is progressing fast and we speculate a future when ultrasound images will be processed free of subjective interpretation. In the meantime, available data demonstrate that a visually calculated LUS score is a useful and easy tool to predict surfactant need in preterm neonates with RDS, to evaluate lung aeration while titrating the respiratory support or to be used as a research outcome measure. ${ }^{75}$ LUS score has its drawbacks (i.e. a semi-quantitative measure, impossibility to detect air-trapping) but is more easy and quickly available at the bedside than more complex techniques, such as electrical impedance tomography or respiratory inductance plethysmography. ${ }^{76,77}$

Semi-quantitative LUS has not only been used for respiratory failure due to a primary pulmonary disorder, but also for neonates with heart defects causing cardiogenic lung oedema. The latter is much more common in adults and, in these cases, the LUS score has been calculated simply by counting B-lines, provided that there was no consolidation due to a parenchymal process. Neonates with congenital heart defects predisposing to pulmonary overflow have a higher B-line count than babies without overflow ${ }^{78}$ and the B-line score also correlates with the duration of ventilation. ${ }^{79}$ Similar applications could be proposed to evaluate pulmonary overflow in the case of haemodynamically significant patent ductus arteriosus or to guide fluid management albeit specific studies are currently lacking.

Standardised protocol for functional LUS: the SAFE algorithm There is a need for specific protocols integrating LUS findings into diagnostic and/or operative flow-charts. Formal LUS protocols for the evaluation of trauma, ${ }^{80}$ dyspnoea $^{81}$ and shock, $^{82}$ are

\section{The SAFE protocol}

This decision tree aims to rapidly rule out the most urgent life threatening emergencies is designed for the neonatologist and need minimal training to perform. in the NICU. The simplified and rapid approach

\section{Emergency ultrasound}

Anterior chest wall substernal or left parasternal axis

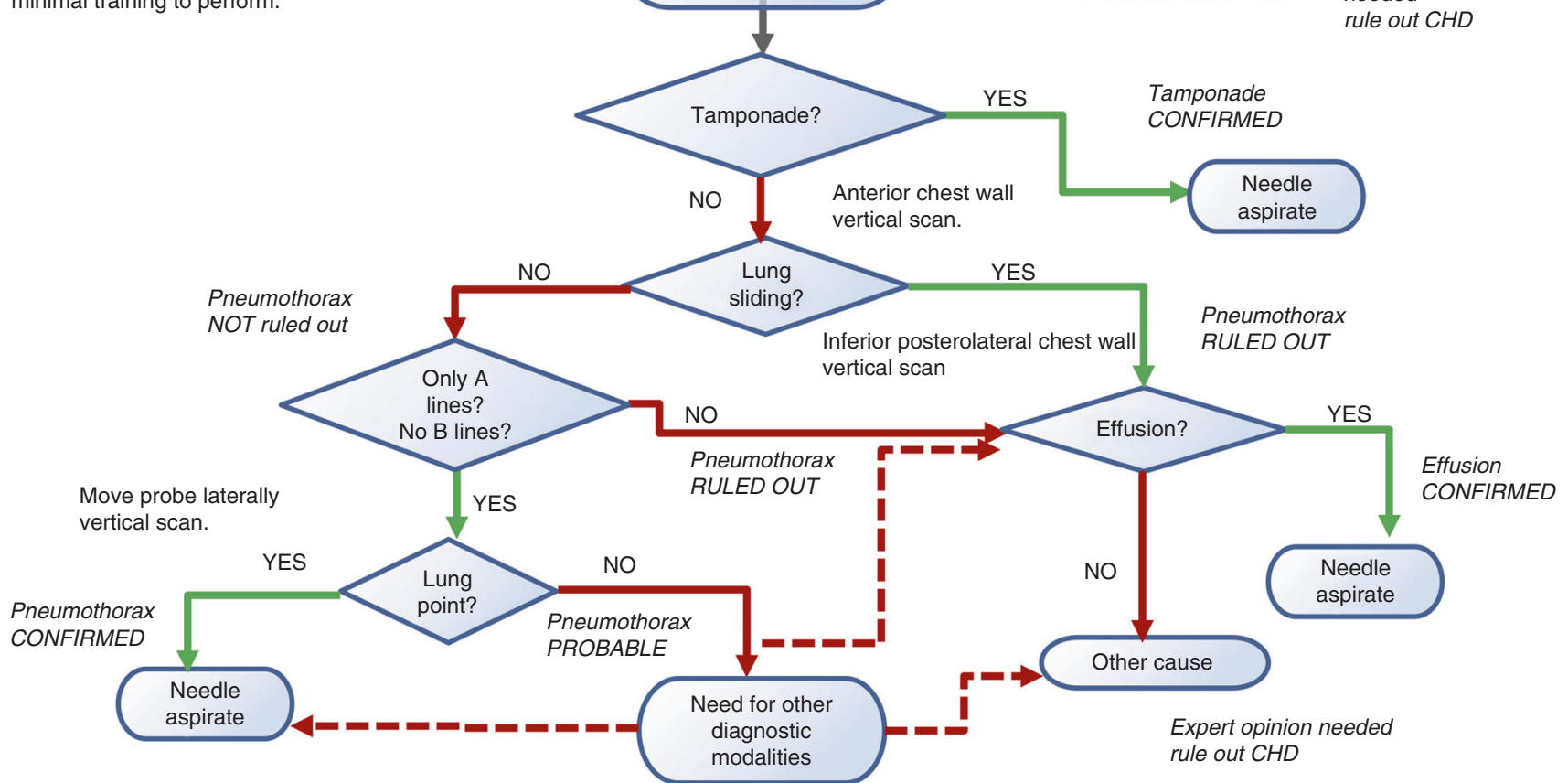

Fig. 3 SAFE (Sonographic Algorithm for liFe threatening Emergencies) algorithm for critically ill neonates. The algorithm is designed for unexpected severe decompensations (bradycardia or severe desaturation requiring resuscitative manoeuvres or significantly increasing oxygen/ventilator parameters to maintain stable oxygen saturation levels) in formerly stable neonates. SAFE protocol starts with a quick 'eyeball' assessment of myocardial contractility (which is accurate enough if there are no arrhythmias, extreme heart rate or ventricular sizes $\left.^{84}\right)$. Then, SAFE screens the more urgent and common causes of life-threatening event: (1) cardiac tamponade, (2) pneumothorax and (3) pleural effusion. The algorithm only takes a few minutes and aims to help diagnosing the most urgent treatable complications whilst awaiting expert help. A paediatric cardiologist evaluation of congenital heart defects is included in the algorithm but only when the most urgent causes have been already ruled out. SAFE is designed for the average neonatologist and may be applied using any probe without losing time to change it 
commonly used in adult critical care. Thus, LUS needs to be integrated into appropriate decision-making algorithms in neonatology. Point-of-care ultrasound is rapidly growing and guidelines about neonatologist-performed echocardiography have been already published, ${ }^{83,84}$ but there are no formal algorithms for the use of LUS in neonatology. A project in this direction has been specifically funded by a European Society for Paediatric Research grant and aimed to create the 'SAFE protocol' (Sonographic Algorithm for liFe threatening Emergencies) to standardise the use of LUS in critically ill neonates. The SAFE protocol is designed for use in the case of unexpected and severe decompensation (bradycardia or severe desaturation requiring resuscitative manoeuvres or significantly increasing oxygen/ ventilator parameters to maintain stable oxygen saturation levels) in formerly stable infants in the NICU. It aims to help the diagnosis of the most urgent treatable complications: current knowledge on the ultrasound detection of the most critical neonatal complications was integrated into the algorithm and rapid diagnosis of unexpected and potentially fatal complications was prioritised. The SAFE protocol starts with an easy, subjective 'eyeball' assessment of myocardial contractility, which is comparable to other techniques which are more accurate but unsuitable during emergencies. $^{85,86}$ Then, SAFE uses standardised items together with a simplified and rapid rule-in/rule-out approach to detect only three main life-threatening complications. As shown in Fig. 3, the ultrasound algorithm is designed by order of urgency. Hence, ruling out cardiac tamponade, which is a rare condition, is the first step in the decision tree since it may be rapidly fatal in the absence of prompt intervention followed by pneumothorax, and lastly, pleural effusion. The latter is also quite unusual, but it is associated to central venous lines often used in NICU care: current guidelines for adult critical care recommend the use of LUS for the diagnosis of pleural effusion, as it outperforms chest X-rays. ${ }^{4}$ SAFE is designed for the average neonatologist, it requires only minimal training and can be performed with a single ultrasound probe, as it targets basic ultrasound signs. A preliminary evaluation of SAFE after basic training in an academic NICU has shown that the algorithm is quick and easy to perform, even for lesserexperienced clinicians. ${ }^{87}$ The SAFE protocol will need to be evaluated prospectively, as it has been done for similar algorithms (Bedside Lung Ultrasound in Emergency (BLUE) and Fluid Administration Limited by Lung Sonography (FALLS)) presently used in adult critical care. ${ }^{80-82}$ Moreover, although any probe can be used, the optimal probe needs to be determined with a specific study. Further work to expand the SAFE protocol to include other organs is ongoing.

\section{LUS-guided procedures}

LUS has been used to guide invasive procedures in order to reduce associated complications. LUS guidance is recommended for chest tube placement in adults, ${ }^{88}$ as this effectively reduces complications. $^{89}$ There are no neonatal studies about these procedures, but it is highly probable that LUS may provide similar advantages, also because of the smaller patients' size. We demonstrated that the LUS detection of tension PNX is extremely accurate and quicker than using conventional radiology. ${ }^{36}$ Other authors successfully performed a LUS-guided drainage of a lifethreatening tension pneumomediastinum. ${ }^{90}$ Even in the absence of specific studies, the use of LUS is advisable in these situations, where enough expertise exists, as LUS will likely assist the operator and make the procedure easier.

LUS has been shown to be effective for verifying endotracheal tube (ETT) position in patients of different ages. This can be achieved by assessing the normal sliding on both hemithoraces that confirms ventilation; in turn, other studies have aimed to directly visualise the ETT tip position. Jaeel et al. recently performed a systematic review of neonatal studies on this topic. ${ }^{91}$ The studies report a successful visualisation of the tube tip in more than $80 \%$ of cases and this correlated with the position observed on chest radiograms in $73-100 \%$ of cases. We must acknowledge that there were variations in techniques, calculations, probes and operators' expertise across the studies: LUS visualisation of ETT does not seem to be straightforward and is potentially subjected to erroneous interpretation. Simpler techniques (such as digital palpation of the ETT tip in the suprasternal notch) have also been proposed. Unless convincing evidence is published, LUS cannot be recommended as a routine technique to verify ETT placement, while end-tidal $\mathrm{CO}_{2}$ measurement is recognised as the gold standard. ${ }^{92}$

LUS is relatively easy to learn in vivo and we have organised successful practical courses since $2014 .{ }^{93}$ However, some interesting bench models have been created to mimic LUS semiology and teach LUS-guided procedures, although they may also be useful as an educational tool for descriptive LUS. Models have been created with plastic phantoms or a wet sponge with or without pork ribs, but also simply using a hand with a wet foam. ${ }^{94-97}$

\section{CONCLUSION}

There is a rapid growth in the use of LUS in neonatology and an increasingly large body of evidence supporting its use in neonatal respiratory care. However, the knowledge available is still far from that acquired in adult critical care. Methodological stringency and multicentre studies are needed. Therefore, we have founded the NeoLUS Group (Neonatal Lung Ultrasound for the neonate and the small infant): a dedicated research network currently counting more than 150 members around the world and disposing of a dedicated page on social networks. ${ }^{98}$ This and other initiatives will contribute to the further development of LUS in neonatology.

\section{ACKNOWLEDGEMENTS}

The authors are grateful to the ESPR Pulmonology Section for their support. The authors are also indebted with Philippe Durand (MD) for the CPAM image. The authors also wish to thank Samsung (Seoul, South Korea) for the technical assistance provided. Videos in the supplementary material have been taken with Samsung HM70A or with General Electrics GE Logiq E9, using a high-frequency linear probe. The development of the SAFE algorithm has been supported by the ESPR Cure \& Care Research Grant 2016 (received by NY).

\section{ADDITIONAL INFORMATION}

The online version of this article (https://doi.org/10.1038/s41390-018-0114-9) contains supplementary material, which is available to authorized users.

Competing interests: The authors declare no competing interests.

Publisher's note: Springer Nature remains neutral with regard to jurisdictional claims in published maps and institutional affiliations.

\section{REFERENCES}

1. Lichtenstein, D. A. \& Menu, Y. A bedside ultrasound sign ruling out pneumothorax in the critically ill. Lung sliding. Chest 108, 1345-1348 (1995).

2. Kurepa, D. et al. Neonatal lung ultrasound exam guidelines. J. Perinatol. 38, 11-22 (2018).

3. Escourrou, G. \& De Luca, D. Lung ultrasound decreased radiation exposure in preterm infants in a neonatal intensive care unit. Acta Paediatr. 105, e237-e239 (2016).

4. International Liaison Committee on Lung Ultrasound (ILC-LUS) for the International Consensus Conference on Lung Ultrasound (ICC-LUS). International evidence-based recommendations for point-of-care lung ultrasound. Intensive Care Med. 38, 577-591 (2012).

5. Lichtenstein, D. et al. The comet-tail artifact. An ultrasound sign of alveolarinterstitial syndrome. Am. J. Respir. Crit. Care Med. 156, 1640-1646 (1997).

6. Volpicelli, G. et al. Lung ultrasound predicts well extravascular lung water but is of limited usefulness in the prediction of wedge pressure. Anesthesiology 121, 320-327 (2014) 
7. Blank, D. A. et al. Lung ultrasound immediately after birth to describe normal neonatal transition: an observational study. Arch. Dis. Child Fetal Neonatal Ed. 103, F157-F162 (2018)

8. Blank, D. A. et al. Lung ultrasound during the initiation of breathing in healthy term and late preterm infants immediately after birth, a prospective, observational study. Resuscitation 114, 59-65 (2017).

9. Martelius, L. et al. Delayed lung liquid absorption after cesarean section at term. Neonatology 104, 133-610 (2013).

10. Copetti, R. \& Cattarossi, L. The 'double lung point': an ultrasound sign diagnostic of transient tachypnea of the newborn. Neonatology 91, 203-209 (2007).

11. Vergine, M. et al. Lung ultrasound accuracy in respiratory distress syndrome and transient tachypnea of the newborn. Neonatology 106, 87-93 (2014).

12. Liu, J. et al. Lung ultrasonography to diagnose transient tachypnea of the newborn. Chest 149, 1269-1275 (2016)

13. Migliaro, F. et al. Is the double lung point an accurate diagnostic marker fir transient tachypnoea of the neonate? A prospective international study. J. Pediatr. Neonatal Individ. Med. 6, e060236 (2017).

14. Raimondi, F. et al. Clinical data are essential to validate lung ultrasound. Chest 149, 1575 (2016).

15. Liu, J. et al. Diagnosis of neonatal transient tachypnea and its differentiation from respiratory distress syndrome using lung ultrasound. Medicine 93, e197 (2014).

16. Copetti, R. et al. Lung ultrasound in respiratory distress syndrome: a useful tool for early diagnosis. Neonatology 94, 52-59 (2008).

17. Rachuri, $\mathrm{H}$. et al. Diagnostic performance of point of care ultrasonography in identifying the etiology of respiratory distress in neonates. Indian J. Pediatr. 84, 267-270 (2017).

18. Brusa, G. et al. Neonatal lung sonography: interobserver agreement between physician interpreters with varying levels of experience. J. Ultrasound Med. 34, 1549-1554 (2014).

19. Sawires, H. K. et al. Use of lung ultrasound in detection of complications of respiratory distress syndrome. Ultrasound Med. Biol. 41, 2319-2325 (2015).

20. Lovrenski, J. Lung ultrasonography of pulmonary complications in preterm infants with respiratory distress syndrome. Ups. J. Med. Sci. 117, 10-17 (2012).

21. Machado, L. U. et al. Surfactant deficiency in transient tachypnea of the newborn. J. Pediatr. 159, 750-754 (2011).

22. Autilo, C. et al. A noninvasive surfactant adsorption test predicting the need for surfactant therapy in preterm infants treated with continuous positive airway pressure. J. Pediatr. 182, 66-73 (2017).

23. Gizzi, C. et al. Continuous positive airway pressure and the burden of care for transient tachypnea of the neonate: retrospective cohort study. Am. J. Perinatol. 32, 939-943 (2015)

24. Cattarossi, L. et al. Surfactant administration for neonatal respiratory distress does not improve lung interstitial fluid clearance: echographic and experimental evidence. J. Perinat. Med. 38, 557-563 (2010).

25. De Luca, D. et al. The Montreux definition of neonatal ARDS: biological and clinical background behind the description of a new entity. Lancet Respir. Med $\mathbf{5}$, 657-666 (2017)

26. Pesenti, A. et al. Imaging in acute respiratory distress syndrome. Intensive Care Med. 42, 686-698 (2016)

27. Riviello, E. D. et al. Hospital incidence and outcomes of the acute respiratory distress syndrome using the Kigali modification of the Berlin definition. Am. J. Respir. Crit. Care Med. 193, 52-59 (2016).

28. De Luca, D. et al. Diagnosis of neonatal ARDS: is Montreux closer to Berlin than to Kigali? Authors' reply. Lancet Respir. Med. 5, e32 (2017).

29. Piastra, M. et al. Lung ultrasound findings in meconium aspiration syndrome. Early Hum. Dev. 90, S41-S43 (2014).

30. Liu, J., Cao, H. Y. \& Fu, W. Lung ultrasonography to diagnose meconium aspiration syndrome of the newborn. J. Int Med Res 44, 1534-1542 (2016).

31. Chen, L. \& Zhang, Z. Bedside ultrasonography for diagnosis of pneumothorax. Quant. Imaging Med. Surg. 5, 618-623 (2015).

32. Alrajab, S. et al. Pleural ultrasonography versus chest radiography for the diagnosis of pneumothorax: review of the literature and meta-analysis. Crit. Care 17, R208 (2013)

33. Migliaro, F. et al. Lung ultrasound-guided emergency pneumothorax needle aspiration in a very preterm infant. BMJ Case Rep. 2014 https://doi.org/10.1136/ bcr-2014-206803 (2014).

34. Cattarossi L., et al. Lung ultrasound diagnostic accuracy in neonatal pneumothorax. Can. Respir. J. 2016, 6515069 (2016).

35. Liu, J. et al. Lung ultrasonography to diagnose pneumothorax of the newborn. Am. J. Emerg. Med. 35, 1298-1302 (2017).

36. Raimondi, F. et al. Lung ultrasound for diagnosing pneumothorax in the critically ill neonate. J. Pediatr. 175, 74-78 (2016).

37. Balcells, C. \& Del Rio, R. Lung ultrasound: an useful tool for the follow-up of neonatal localized interstitial emphysema. J. Pediatr. 166, 1543 (2015).
38. Saracino, C. \& Tessaro, M. Pneumomediastinum as a sonographic mimic of pneumothorax. J. Ultrasound Med. 34, 1521-1522 (2015).

39. Matsumoto, T. \& Matano, H. The still lung point: new sonographic evidence for pneumomediastinum. Am. J. Emerg. Med. 34, 344.e1-2 (2016).

40. Liu, J. et al. Lung ultrasonography for the diagnosis of severe neonatal pneumonia. Chest 146, 383-388 (2014).

41. Chen, S. W. et al. Routine application of lung ultrasonography in the neonatal intensive care unit. Medicine 96, e5826 (2017).

42. Pereda, M. A. et al. Lung ultrasound for the diagnosis of pneumonia in children: a meta-analysis. Pediatrics 135, 714-722 (2015).

43. Alzahrani, S. A. et al. Systematic review and meta-analysis for the use of ultrasound versus radiology in diagnosing of pneumonia. Crit. Ultrasound J. 9, 6 (2017).

44. Long, L. et al. Lung ultrasound for the diagnosis of pneumonia in adults: a metaanalysis. Medicine 96, e5713 (2017)

45. Staub, L. J., Biscaro, R. R. M. \& Maurici, R. Accuracy and applications of lung ultrasound to diagnose ventilator-associated pneumonia: a systematic review. J. Intensive Care Med. 33, 447-455 (2018).

46. Caiulo, V. A. et al. Lung ultrasound in bronchiolitis: comparison with chest X-ray. Eur. J. Pediatr. 170, 1427-1433 (2011).

47. Taveira, M. et al. Can a simple lung ultrasound score predict length of ventilation for infants with severe acute viral bronchiolitis? Arch. Pediatr. 25, 112-117 (2018).

48. Hammer, J., Numa, A. \& Newth, C. J. L. Acute respiratory distress syndrome caused by respiratory syncytial virus. Pediatr. Pulmonol. 23, 176-183 (1997).

49. Varshney, T. et al. Point-of-care lung ultrasound in young children with respiratory tract infections and wheeze. Emerg. Med J. 33, 603-610 (2016).

50. Basile, V. et al. Lung ultrasound: a useful tool in diagnosis and management of bronchiolitis. BMC Pediatr. 15, 63 (2015).

51. Yousef, N. \& De Luca, D. The role of lung ultrasound in viral lower respiratory tract infections. Am. J. Perinatol. 35, 527-529 (2018).

52. Tsung, J. W., Kessler, D. O. \& Shah, V. P. Prospective application of clinicianperformed lung ultrasonography during the 2009 H1N1 influenza A pandemic: distinguishing viral from bacterial pneumonia. Crit. Ultrasound J. 4, 16 (2012)

53. Shen, P. et al. Dynamic assessment of lung injury by ultrasound in a case with H7N9 influenza. Crit. Care 17, 438 (2013).

54. Testa, A., Soldati, G. \& Copetti, R. Early recognition of the 2009 pandemic influenza A (H1N1) pneumonia by chest ultrasound. Crit. Care 16, R30 (2012).

55. Volpicelli, G. \& Frascisco, M. F. Sonographic detection of radio-occult interstitial lung involvement in measles pneumonitis. Am. J. Emerg. Med. 27, 128.e1-3 (2009).

56. Ammari, A. et al. Variables associated with the early failure of nasal CPAP in very low birth weight infants. J. Pediatr. 147, 341-347 (2005).

57. Moya, M. P. et al. Reliability of CXR for the diagnosis of bronchopulmonary dysplasia. Pediatr. Radiol. 31, 339-342 (2001).

58. Avni, E. F. et al. Sonographic prediction of chronic lung disease in the premature undergoing mechanical ventilation. Pediatr. Radiol. 26, 463-469 (1996).

59. Pieper, C. H., Smith, J. \& Brand, E. J. The value of ultrasound examination of the lungs in predicting bronchopulmonary dysplasia. Pediatr. Radiol. 34, 227-231 (2004).

60. Steinhorn, R. et al. Chronic pulmonary insufficiency of prematurity: developing optimal endpoints for drug development. J. Pediatr. 191, 15-21.e1 (2017).

61. David, M., Lamas-Pinheiro, R. \& Henriques-Coelho, T. Prenatal and postnatal management of congenital pulmonary airway malformation. Neonatology 110, 101-115 (2016)

62. Yousef, N. et al. Lung ultrasound findings in congenital pulmonary airway malformations. Am. J. Perinatol. (2018) https://doi.org/10.1055/s-0038-1645861. (Epub ahead of print).

63. Lichtenstein, D. A. BLUE-protocol and FALLS-protocol: two applications of lung ultrasound in the critically ill. Chest 147, 1659-1670 (2015).

64. Corradi, F., Brusasco, C. \& Pelosi, P. Chest ultrasound in acute respiratory distress syndrome. Curr. Opin. Crit. Care 20, 98-103 (2014).

65. Raimondi, F. et al. Can neonatal lung ultrasound monitor fluid clearance and predict the need of respiratory support? Crit. Care 16, R220 (2012).

66. Raimondi, F. et al. Use of neonatal chest ultrasound to predict noninvasive ventilation failure. Pediatrics 134, e1089-e1094 (2014)

67. Rodríguez-Fanjul, J. et al. Lung ultrasound as a predictor of mechanical ventilation in neonates older than 32 weeks. Neonatology 110, 198-203 (2016).

68. Bouhemad, B. et al. Bedside ultrasound assessment of positive end-expiratory pressure-induced lung recruitment. Am. J. Respir. Crit. Care Med. 183, 341-347 (2011).

69. Via, G. et al. Lung ultrasound in the ICU: from diagnostic instrument to respiratory monitoring tool. Minerva Anestesiol. 78, 1282-1296 (2012).

70. Brat, R. et al. Lung ultrasonography score to evaluate oxygenation and surfactant need in neonates treated with continuous positive airway pressure. JAMA Pediatr. 169, e151797 (2015). 
71. De Martino L., et al. Lung ultrasound score predicts surfactant need in extremely preterm neonates. Pediatrics (2018) Accepted in press https://doi.org/10.1542/ peds.2008-1536

72. Veeramani, S. K. \& Muthusamy, E. Detection of abnormalities in ultrasound lung image using multi-level RVM classification. J. Matern Fetal Neonatal Med 29, 1844-1852 (2016).

73. Palacio, M. et al. Fetal Lung Texture Team. Prediction of neonatal respiratory morbidity by quantitative ultrasound lung texture analysis: a multicenter study. Am. J. Obstet. Gynecol. 217, 196.e1-196 (2017).

74. Raimondi, F. et al. Can we assess the severity of neonatal respiratory distress by ultrasound? A comparison of three methods. J. Pediatr. Neonatal Individ. Med 6, e060236 (2017).

75. De Luca, D. Noninvasive high-frequency ventilation and the errors from the past: designing simple trials neglecting complex respiratory physiology. J. Perinatol. 37, 1065-1066 (2017).

76. Frerichs, I. et al. Chest electrical impedance tomography examination, data analysis, terminology, clinical use and recommendations: consensus statement of the TRanslational EIT developmeNt stuDy group. Thorax 72, 83-93 (2017).

77. Reiterer, F., Sivieri, E. \& Abbasi, S. Evaluation of bedside pulmonary function in the neonate: from the past to the future. Pediatr. Pulmonol. 50, 1039-1050 (2015).

78. Rodríguez-Fanjul, J. et al. Usefulness of lung ultrasound in neonatal congenital heart disease (LUSNEHDI): lung ultrasound to assess pulmonary overflow in neonatal congenital heart disease. Pediatr. Cardiol. 37, 1482-1487 (2016).

79. Kaskinen, A. K. et al. Assessment of extravascular lung water by ultrasound after congenital cardiac surgery: lung ultrasound after congenital cardiac surgery. Pediatr. Pulmonol. 52, 345-352 (2017).

80. Scalea, T. M. et al. Focused assessment with sonography for trauma (FAST): results from an international consensus statement. J. Trauma 46, 492-298 (1999).

81. Lichtenstein, D. \& Meziere, G. A. Relevance of lung ultrasound in the diagnosis of acute respiratory failure: the BLUE protocol. Chest 134, 117-125 (2008).

82. Atkinson, $\mathrm{P}$. et al. International federation for emergency medicine consensus statement: sonography in hypotension and cardiac arrest $(\mathrm{SHoC})$ : an international consensus on the use of point of care ultrasound for undifferentiated hypotension and during cardiac arrest. CJEM 19, 459-470 (2017).

83. Mertens, L. et al. Targeted neonatal echocardiography in the neonatal intensive care unit: practice guidelines and recommendations for training. Writing Group of the American Society of Echocardiography (ASE) in collaboration with the European Association of Echocardiography (EAE) and the Association for European Pediatric Cardiologists (AEPC). J. Am. Soc. Echocardiogr. 24, 1057-1078 (2011).
84. de Boode, W. P. et al. Recommendations for neonatologist performed echocardiography in Europe: Consensus Statement endorsed by European Society for Paediatric Research (ESPR) and European Society for Neonatology (ESN). Pediatr. Res. 80, 465-471 (2016)

85. Marwick, T. H. Techniques for comprehensive two dimensional echocardiographic assessment of left ventricular systolic function. Heart $\mathbf{8 9}$ (Suppl 3), iii2-8 (2003).

86. Foster, E. \& Cahalan, M. K. The search for intelligent quantitation in echocardiography: "eyeball," "trackball" and beyond. J. Am. Coll. Cardiol. 22, 848-850 (1993).

87. Yousef, N., Shankar-Aguilera, S. \& De Luca, D. The SAFE protocol: a sonographic algorithm for life-threatening emergencies in the neonatal intensive care unit. $J$. Pediatr. Neonatal Individ. Med. 6, e060236 (2017).

88. Dancel, R. et al. Recommendations on the use of ultrasound guidance for adult thoracentesis: a position statement of the society of hospital medicine. J. Hosp. Med. 13, 126-135 (2018).

89. Jones, P. W. et al. Ultrasound-guided thoracentesis: is it a safer method? Chest 123, 418-423 (2003).

90. Mohamed, I. S. et al. Ultrasound guided percutaneous relief of tension pneumomediastinum in a 1-day-old newborn. Arch. Dis. Child Fetal Neonatal Ed. 92 F458 (2007).

91. Jaeel, P., Sheth, M. \& Nguyen, J. Ultrasonography for endotracheal tube position in infants and children. Eur. J. Pediatr. 176, 293-300 (2017).

92. Hosono, S. et al. A role of end-tidal $\mathrm{CO}(2)$ monitoring for assessment of trachea intubations in very low birth weight infants during neonatal resuscitation at birth J. Perinat. Med. 37, 79-88 (2009).

93. https://www.facebook.com/LUSPEDSchool/ (accessed 29 April 2018).

94. Rippey, J. \& Gawthrope, I. Creating thoracic phantoms for diagnostic and procedural ultrasound training. Australas. J. Ultrasound Med 15, 43-54 (2012).

95. Vetrugno, L. et al. Phantom model and scoring system to assess ability in ultrasound-guided chest drain positioning. Crit. Ultrasound J. 8, 1 (2016).

96. Park, E. J., Yoon, Y. T., Hong, C. K., Ha, Y. R. \& Ahn, J. H. Randomized, noninferiority study between video versus hand ultrasound with wet foam dressing materials to simulate B-lines in lung ultrasound: a CONSORT-compliant article. Medicine $\mathbf{9 6}$ e7642 (2017).

97. Blüthgen, C., Sanabria, S., Frauenfelder, T., Klingmüller, V. \& Rominger, M. Economical sponge phantom for teaching, understanding, and researching $\mathrm{A}$ - and Bline reverberation artifacts in lung ultrasound. J. Ultrasound Med. 36, 2133-2142 (2017).

98. https://www.facebook.com/groups/1493243264284547/ (accessed 1 Mar 2018). 